\begin{tabular}{|c|l|}
\hline Title & Surface spin polarization of the nonstoichiometric Heusler all loy Co2MnSi \\
\hline Author(s) & $\begin{array}{l}\text { Wüstenberg, Jan-Peter; Fetzer, Roman; A eschlimann, Martin; Cinchetti, Mirko; Minár, Jan; Braun, Jürgen; Ebert, } \\
\text { Hubert; Ishikawa, Takay uki; Uemura, Tetsuy a; Y amamoto, Masafumi }\end{array}$ \\
\hline Citation & $\begin{array}{l}\text { Physical Review B, 85(6), 064407 } \\
\text { https://doi.org/10.1103/PhysRevB.85.064407 }\end{array}$ \\
\hline Issue Date & 2012-02-01 \\
\hline Doc URL & http://hdl.handle.net/2115/48550 \\
\hline Rights & O2012A A merican Physical Society \\
\hline Type & article \\
\hline File Information & PRB85-6_064407.pdf \\
\hline
\end{tabular}

Instructions for use 


\title{
Surface spin polarization of the nonstoichiometric Heusler alloy $\mathrm{Co}_{2} \mathrm{MnSi}$
}

\author{
Jan-Peter Wüstenberg, ${ }^{*}$ Roman Fetzer, Martin Aeschlimann, and Mirko Cinchetti \\ Department of Physics and Research Center OPTIMAS, University of Kaiserslautern, Erwin-Schrödingerstr. 46, \\ DE-67663 Kaiserslautern, Germany
}

\author{
Jan Minár, Jürgen Braun, and Hubert Ebert \\ Department Chemie, Ludwig-Maximilians-Universität München, Butenandtstraße 11, DE-81377 München, Germany
}

Takayuki Ishikawa, Tetsuya Uemura, and Masafumi Yamamoto

Division of Electronics for Informatics, Hokkaido University, Kita 14 Nishi 9, Sapporo 060-0814, Japan

(Received 28 October 2011; revised manuscript received 21 December 2011; published 15 February 2012)

\begin{abstract}
Using a combined approach of spin-resolved photoemission spectroscopy, band structure and photoemission calculations we investigate the influence of bulk defects and surface states on the spin polarization of nonstoichiometric $\mathrm{Co}_{2} \mathrm{Mn}_{\alpha} \mathrm{Si}$ thin films (with $\alpha=0.69$ and $\alpha=1.19$ ) with bulk $L 2_{1}$ order. We find that for Mn-poor alloys the spin polarization at the Fermi energy $\left(E_{F}\right)$ is negative due to the presence of $\mathrm{Co}_{\mathrm{Mn}}$ antisite and minority surface state contributions. In Mn-rich alloys, the suppression of $\mathrm{Co}_{\mathrm{Mn}}$ antisites leads to a positive spin polarization at $E_{F}$, and the influence of minority surface states on the photoelectron spin polarization is reduced.
\end{abstract}

DOI: 10.1103/PhysRevB.85.064407

PACS number(s): 75.70.Rf, 75.50.Cc, 73.20.-r, 71.15.-m

\section{INTRODUCTION}

Heusler compounds are intermetallic compounds with the composition $X_{2} Y Z$, crystallizing in the $L 2_{1}$ structure. $X$ and $Y$ atoms are transition metals and $Z$ is a main group element. Due to a high Curie temperature and predicted full conduction electron spin polarization, some members of the familiy of Heusler compounds are expected to improve significantly the performance of magnetic tunneling junctions (MTJs) based on the tunneling magnetoresistance effect. ${ }^{1}$

Among the class of ferromagnetic Heusler alloys, $\mathrm{Co}_{2} \mathrm{MnSi}$ (CMS) has been most successfully applied in MTJs. ${ }^{2,3}$ It is predicted to be a half-metallic ferromagnet with a large magnetic moment of $5 \mu_{B}$ per formula unit and a high Curie temperature of $985 \mathrm{~K}^{4-7}$ All calculations predict a nearly half-metallic behavior of bulk single crystals owing to a gap in the minority density of states around the Fermi energy, at least at low temperature. In contrast to the large bulk band gaps predicted by density functional theory (DFT) calculations (cf. Ref. 5) the experimental width of this band gap in thin film structures has been found to be about $0.4 \mathrm{eV}$ using tunneling spectroscopy at interfaces with different barrier materials. ${ }^{8,9}$

In order to maintain a high electron spin polarization even at room temperature, the Fermi energy should lie preferentially in the middle of the half-metallic gap. In this aspect, experiments and theory disagree considerably. While DFT calculations find the Fermi energy in CMS to be situated close to the minority valence band, MTJs using $\mathrm{MgO}$ and $\mathrm{AlO}_{x}$ barriers indicate the Fermi energy around midgap ${ }^{9}$ and $10 \mathrm{meV}$ below the conduction band, ${ }^{8}$ respectively.

So far, experimental spin-polarization values have not reached the ideal limit of $100 \%$ at the Fermi energy. In most publications, an effective spin polarization is inferred from the tunnel magnetoresistance ratio (TMR) of MTJ by making use of the Jullière formula TMR $=2 P_{\mathrm{J} 1} P_{\mathrm{J} 2} /\left(1-P_{\mathrm{J} 1} P_{\mathrm{J} 2}\right)$, where $P_{\mathrm{J} 1}$ and $P_{\mathrm{J} 2}$ denote the effective spin-polarization values for the respective ferromagnet/insulator interface. ${ }^{10}$
A high tunneling spin polarization of $P_{\mathrm{J}}=89 \%$ has been inferred using the tunneling magneto-resistance ratio (TMR) of a CMS/AlOx/CMS MTJ at $2 \mathrm{~K}$, decreasing to $72 \%$ at room temperature. ${ }^{8}$ Point contact Andreev reflection (PCAR) measurements at $T=4.2 \mathrm{~K}$ on bulk single crystals revealed $P_{\mathrm{PCAR}}=59 \%$. $^{11}$ Using spin-resolved photoelectron spectroscopy (SR-PES) carried out at room temperature, a maximum surface spin polarization value of $P_{\mathrm{PES}}=12 \%$ was obtained for thin films of $\mathrm{Co}_{2} \mathrm{MnSi}$ (100) grown on GaAs(001) using $70 \mathrm{eV}$ photons. ${ }^{12}$ Among Heusler compounds grown on $\mathrm{MgO}(100)$ substrates, $\mathrm{Co}_{2} \mathrm{FeSi}, \mathrm{Co}_{2} \mathrm{MnGa}$, and $\mathrm{Co}_{2} \mathrm{Cr}_{0.6} \mathrm{Fe}_{0.4} \mathrm{Al}$ were investigated by SR-PES so far. ${ }^{13-17}$

The strong temperature dependence of the TMR of Heusler based MTJs has been related to a temperature-dependent spin polarization in the ferromagnetic electrode via the Jullière formula. Most prominently, the so-called nonquasiparticle states have been invoked. ${ }^{6,18}$ However, high-energy photoemission experiments, applied to verify spin integrated calculated valence band densities of states could not confirm the predicted changes in the peak positions. ${ }^{19,20}$ Other intrinsic bulk depolarization mechanisms such as spin-orbit interaction, ${ }^{21}$ magnetic sublattice noncollinearity, fluctuation-induced hybridization changes, and weakened exchange coupling of the magnetic surface layer have been discussed, ${ }^{22,23}$ but still lack experimental verification.

Extrinsic effects such as deviations of the $L 2_{1}$ structure due to disorder and nonstoichiometric composition can lead to additional electronic states in the minority gap that reduce the spin polarization at the Fermi energy. Opposite to various noncritical types of defects, $\mathrm{Co}_{\mathrm{Mn}}$ antisite defects have been predicted to induce minority defect states at the Fermi energy. ${ }^{24-26}$ Recent results suggest that such antisites are hard to avoid in stoichiometric thin films but can at least partly be compensated by increasing the $\mathrm{Mn} / \mathrm{Co}$ ratio. ${ }^{2,27}$ However, at large Mn concentrations, the precipitation of nonferromagnetic $\mathrm{Mn}_{3} \mathrm{Si}$ clusters has been postulated. ${ }^{26}$ 
Additional spin-polarized electronic states may arise at surfaces and interfaces due to hybridization changes that occur at the boundary atomic layers. ${ }^{28}$ Such states are well known for surfaces with a gap in the surface-projected electronic band structure, for example, in semiconductors or in the projected bulk band gaps at the low index surfaces of various transition metal surfaces. ${ }^{28}$ Such localized states can contribute significantly to the electronic transport properties of tunneling magnetoresistive devices ${ }^{29}$ and have been used to explain the $I / V$ characteristics of magnetic tunneling junctions. ${ }^{9}$

For free $\mathrm{Co}_{2} \mathrm{MnSi}$ (CMS)(100) surfaces, ab initio atomistic thermodynamics predict four stable terminations: Mn-Mn, $\mathrm{Mn}-\mathrm{Si}, \mathrm{Si}-\mathrm{Si}$, and vacancy-Si. Apart from the Mn-Mn termination, all stable surface configurations have been predicted to reduce the surface spin polarization due to the formation of minority surface state bands that are derived from partly filled Co $d_{3 z^{2}-r^{2}}$ orbitals and cross the Fermi level within the bulk minority band gap. ${ }^{30}$

In this paper, we present a systematic characterization of the free (100) surface of Mn-deficient and Mn-rich CMS films in terms of geometric and spin-resolved electronic structure. The low-energy electron diffraction (LEED) patterns indicate a well ordered surface. Using low-energy SR-PES, the spin-resolved electronic structure is determined and compared to state of the art photoemission calculations performed for different surface terminations and including dynamic correlation effects. We find that both LEED patterns and spin-resolved spectra corroborate the assumption of a $\mathrm{Mn}-\mathrm{Si}$ or vacancy-Si terminated surface, in particular, for the Mndeficient phase. We find a negative spin polarization at $E_{F}$ for $\mathrm{Mn}$-poor alloys due to the presence of $\mathrm{Co}_{\mathrm{Mn}}$ antisites and minority-surface-state contributions. In Mn-rich alloys, the suppression of $\mathrm{Co}_{\mathrm{Mn}}$ antisites leads to a positive spin polarization at the Fermi energy, and the influence of minority surface states on the photoelectron spin polarization is reduced.

\section{CALCUlational DETAILS}

To fully understand the experimental data, we have performed first-principles calculations using the local-spindensity approximation (LSDA) of density functional theory within the spin-polarized fully relativistic Korringa-KohnRostoker Green's function method (SPR-KKR). ${ }^{31-33}$ For the exchange and correlation potential we applied the Vosko, Wilk, and Nusair parametrization. ${ }^{34}$ To account for electronic correlations beyond the LSDA, we employed a combined LSDA plus dynamical mean-field theory (LSDA + DMFT) scheme, self-consistent in both the self-energy calculation and in the charge density calculation, as implemented within the relativistic SPR-KKR formalism. ${ }^{35,36}$ As a DMFT solver, the relativistic version of the so-called spin-polarized $T$ matrix plus fluctuation exchange (SPTF) approximation ${ }^{37,38}$ was used. In contrast to most other LSDA + DMFT implementations, within the SPR-KKR scheme the complex and energy-dependent self-energy $\Sigma_{\text {DMFT }}$ is implemented as an additional energy-dependent potential to the radial Dirac equation, which is solved in order to calculate the new Green's function. This procedure is repeated until self-consistency in both the self-energy and the charge density is achieved. The double counting problem (separation of the Hubbard
Hamiltonian from the LSDA one) was considered within the usual around atomic limit (AAL). This scheme was successfully used before in describing magnetic properties of CMS. ${ }^{5}$ An appealing feature of the multiple scattering formalism is the possibility to calculate substitutionally disordered materials within the coherent potential approximation (CPA). The CPA is considered to be the best theory among the so-called single-site (local) alloy theories that assume complete random disorder and ignore short-range order. A combination of the CPA and LSDA + DMFT within the SPR-KKR method has been used recently. ${ }^{36,39,40}$

The self-energy within the DMFT is parametrized by the average screened Coulomb interaction $U$ and the Hund exchange interaction $J$. The $J$ parameter can be calculated directly within the LSDA and is approximately the same for all $3 d$ elements; we used $J_{\mathrm{Mn} \text {,Co }}=0.9 \mathrm{eV}$ for the $\mathrm{Mn}$ and Co atoms throughout our work. The parameter $U$ is strongly affected by the metallic screening and it is estimated for the $3 d$ metals between $1-3 \mathrm{eV}$. We used $U_{\mathrm{Mn}, \mathrm{Co}}=2.3 \mathrm{eV}$ for the $\mathrm{Mn}$ and Co atoms. ${ }^{5}$ DMFT calculations have been performed for $T=400 \mathrm{~K}$ and we used 4096 Matsubara poles to calculate the corresponding SPTF self-energy. The effective potentials were treated within the atomic sphere approximation (ASA).

As a first step of our theoretical investigations, we performed LSDA + DMFT self-consistent electronic structure calculations for ordered bulk $\mathrm{Co}_{2} \mathrm{MnSi}$ and $\mathrm{CPA}$ calculations for three different disordered compositions: $\mathrm{Co}_{2}\left(\mathrm{Mn}_{0.69}\right.$ Vacancy $\left._{0.31}\right) \mathrm{Si}, \mathrm{Co}_{2}\left(\mathrm{Co}_{0.168} \mathrm{Mn}_{0.748} \mathrm{Si}_{0.084}\right) \mathrm{Si}$ (corresponding to a Mn-poor compound of nominal $\mathrm{Co}_{2} \mathrm{Mn}_{0.69} \mathrm{Si}$ composition), and $\left(\mathrm{Co}_{1.909} \mathrm{Mn}_{0.091}\right) \mathrm{Mn}_{1.0}\left(\mathrm{Si}_{0.955} \mathrm{Mn}_{0.045}\right)$ (corresponding to a Mn-rich compound of nominal $\mathrm{Co}_{2} \mathrm{Mn}_{1.19} \mathrm{Si}$ composition). For all systems we used the $L 2_{1}$ structure with the experimental lattice constant $\left(a_{\text {lat }}=5.654 \AA\right)$. In addition, to study surface effects for the free $\mathrm{Co}_{2} \mathrm{MnSi}(100)$ surface, semi-infinite screened KKR calculations ${ }^{31}$ (i.e., without using artificial slab geometry) have been performed for four stable terminations: $\mathrm{Mn}-\mathrm{Mn}, \mathrm{Mn}-\mathrm{Si}, \mathrm{Si}-\mathrm{Si}$, and vacancy-Si. For the multipole expansion of the Green's function, an angular momentum cutoff of $l_{\max }=3$ was used. The integration in the $\boldsymbol{k}$ space was performed by the special points method using $1600 k$ points in the irreducible wedge.

As a second step, actual valence band photoemission spectra were calculated using a recent implementation of the fully relativistic CPA formalism within the LSDA + DMFT method in the framework of the one step model of photoemission, which implicitly includes all matrix elements and surface effects ${ }^{41,42}$ and therefore allows for a direct comparison to the corresponding experimental data.

\section{EXPERIMENT}

We have investigated two different $\mathrm{Co}_{2} \mathrm{Mn}_{\alpha} \mathrm{Si}(100)$ surfaces. Both films showed $L 2_{1}$ bulk order in the XRD patterns. Sample CMS069 with $\alpha=0.69$ exhibited a nominal bulk $\mathrm{Co}_{2} \mathrm{Mn}_{0.69} \mathrm{Si}_{1.01}$ composition, while sample CMS119 with $\alpha=1.19$ exhibited $\mathrm{Co}_{2} \mathrm{Mn}_{1.19} \mathrm{Si}_{0.88}$ composition. The layer structure of the samples was as follows: (from the substrate side) $\mathrm{MgO}$ buffer $(10 \mathrm{~nm}) / \mathrm{CMS}(\alpha) / \mathrm{MgO}$ barrier $(2 \mathrm{~nm})$, grown on a $\mathrm{MgO}(100)$ substrate. The CMS thickness was $50 \mathrm{~nm}$ for CMS069 and $30 \mathrm{~nm}$ for CMS119. Each layer was 
successively deposited in an ultrahigh vacuum chamber (with a base pressure of about $6 \times 10^{-10}$ mbar). The CMS layer was deposited at room temperature (RT) using magnetron sputtering and subsequently annealed in situ at $600^{\circ} \mathrm{C}$. The $\mathrm{MgO}(100)$ barrier was deposited at RT by electron beam evaporation. Each layer in the above layer structure was grown epitaxially. ${ }^{27}$ Before LEED observations or spin-resolved photoemission measurements, samples were annealed to $500{ }^{\circ} \mathrm{C}$ in situ in an ultrahigh vacuum chamber for observations and measurements. All measurements were carried out at room temperature.

LEED patterns of the sample surfaces were obtained by means of an Omicron 3-grid SpectaLEED system after a cleaning procedure, consisting of the removal of the $\mathrm{MgO}$ barrier by $500 \mathrm{eV} \mathrm{Ar}^{+}$sputtering and subsequent annealing of the CMS samples to $500^{\circ} \mathrm{C}$. From the ratio of spot width (Lorentz FWHM) and reciprocal lattice vector, an instrumental transfer width ${ }^{43}$ of at least $12 \mathrm{~nm}$ was determined at a clean $\mathrm{Cu}(100)$ reference surface and at $E=80 \mathrm{eV}$. The chemical composition was investigated using an Auger system based on a cylindrical mirror energy analyzer, manufactured by Omicron NanoTechnology. Relative composition values were obtained using the method described in Ref. 44.

The photoemission spectra were obtained using the linearly polarized 4th harmonic (photon energy $5.9 \mathrm{eV}$ ) of a $100 \mathrm{fs}$ Ti:Sapphire oscillator (Spectra Physics Tsunami). Using a phase retarding plate the light polarization could be switched from $s$ polarization (electrical field vector perpendicular to the plane of incidence) to $p$ polarization (electrical field vector within the plane of incidence). The photon angle of incidence onto the samples was $45^{\circ}$. The photoelectron spectra were taken in normal emission geometry, integrating along the $\Gamma \mathrm{X}$ momentum direction. A biasing voltage of $-4 \mathrm{~V}$ was applied between sample and detector in order to increase the effective parallel momentum integration window. Due to the large unit cell size of CMS and the large angular acceptance angle of the analyzer of $\pm 15^{\circ}$ along the sample [110] direction $(\Gamma \mathrm{K}=\bar{\Gamma} \overline{\mathrm{X}}$ momentum direction), the photoemission spectrum integrates electron momenta over almost $60 \%$ of the surface Brillouin zone of $\mathrm{Co}_{2} \mathrm{MnSi}$ in $\Gamma \mathrm{K}$ direction.

Spin-polarized photoemission spectra were recorded by means of a commercial $90^{\circ}$ cylindrical sector energy analyzer (Focus CSA 300), equipped with an additional spin detector based on spin-polarized LEED at a W(100) crystal (Focus SPLEED). The achieved effective energy resolution is $210 \mathrm{meV}$ full width at half maximum as determined from a simulation of the work function cutoff of the spectra.

Due to the detection geometry, the electron spin polarization (ESP) along the CMS [011] direction (in-plane) and along the surface normal (out-of-plane) can be determined. A Sherman factor of $S=0.2$ was used to determine the spin polarization from the measured intensity asymmetry of opposite diffraction spots. Apart from statistical errors, a systematic relative spin polarization error due to uncertainties in the determination of the Sherman factor $(S=0.2, \ldots, 0.25)$ can not be excluded. ${ }^{45}$ Minority and majority spin spectra were calculated using the formula $I_{\uparrow \downarrow}=\bar{I}(1 \pm P)$ using the experimental ESP $P$ and the averaged count rate $\bar{I}$ of corresponding opposite channeltrons. For the spin-resolved measurements, the CMS film was magnetized in remanence along the in-plane [011] direction by applying an external in-plane magnetic field of $\mu_{0} H=15 \mathrm{mT}$, which is sufficient to saturate the magnetization of the investigated samples. Detector-related asymmetries are canceled out by taking separate measurements with reversed sample magnetization. With this setup, an in-plane spin polarization of $30 \%$ at the Fermi energy was achieved on a 200-nm thick film of polycrystalline $\mathrm{Co}_{70} \mathrm{Fe}_{30}$. The out-of-plane spin component vanished within the experimental error.

\section{SURFACE CHARACTERIZATION}

Before investigating the surface electronic structure of $\mathrm{Co}_{2} \mathrm{MnSi}(100)$, a clean and well defined sample surface was prepared. This was accomplished by sputter cleaning using 500-eV Ar ${ }^{+}$ions and subsequent heating of the sample. The surface quality was confirmed by Auger spectroscopy and by LEED pattern analysis.

In Fig. 1, the LEED patterns of an $\mathrm{MgO}$ tunneling barrier (B1 structure), sample CMS069, and $\mathrm{Co}_{2} \mathrm{Cr}_{0.6} \mathrm{Fe}_{0.4} \mathrm{Al}$ (B2 structure $^{16,46}$ ) are shown. The pattern of CMS069 and CMS119 (not shown) are identical. Sharp spots indicate a well ordered CMS surface. Comparing with the $\mathrm{MgO}(100)$ surface pattern, we observe the compressed and rotated spot pattern expected from the larger unit cell of CMS and the resulting $45^{\circ}$ growth in order to minimize lattice misfit. The additional (11) edge spots with respect to the B2 pattern indicate the existence of square two-atomic surface lattices. We therefore conclude that the outermost surface layers show preferentially $L 2_{1}$ ordering. Furthermore, this pattern is expected for the energetically favored $\mathrm{Mn}-\mathrm{Si}$ (or vacancy-Si) terminations as reported earlier for stoichiometric CMS. ${ }^{12,30,47}$ Nevertheless, other terminations or mixtures of them can not be completely excluded.

In order to determine the chemical composition of the CMS069 surface, Auger spectroscopy has been carried out. The obtained spectrum of sample CMS069 is displayed in Fig. 2. It clearly shows the signatures of the constituting elements, along with some residual argon from the sputtering process, carbon $(1 \%)$ and oxygen $(3 \%)$, which could not be removed without leaving the optimum annealing temperature range. Using the peak-to-peak amplitudes of the differential spectrum and the sensitivity factors given in Ref. 48, the composition of the surface was obtained. The analysis of the differentiated Auger spectrum yields a nominal surface composition of $\mathrm{Co}_{2} \mathrm{Mn}_{0.85} \mathrm{Si}_{0.93}$, thus a slightly higher $\mathrm{Mn}$ content than the thin film value determined by ICP analysis. ${ }^{27}$ For CMS119, we obtain a nominal surface composition of $\mathrm{Co}_{2} \mathrm{Mn}_{1.19} \mathrm{Si}_{1.35}$.
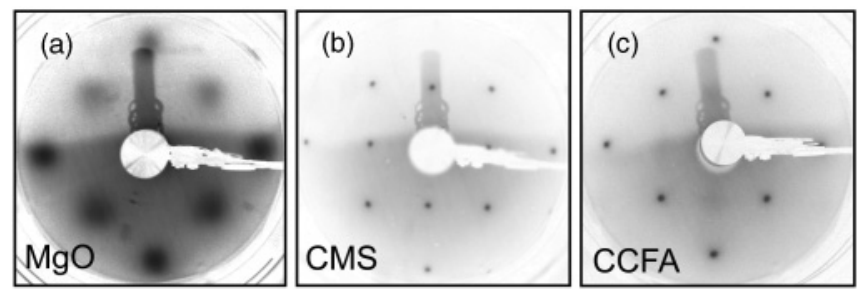

FIG. 1. LEED patterns of (a) a $\mathrm{MgO}$ barrier $(E=82 \mathrm{eV})$, (b) sample CMS069 ( $E=57 \mathrm{eV})$, and (c) $\mathrm{Co}_{2} \mathrm{Cr}_{0.6} \mathrm{Fe}_{0.4} \mathrm{Al}(E=$ $74 \mathrm{eV})$. 


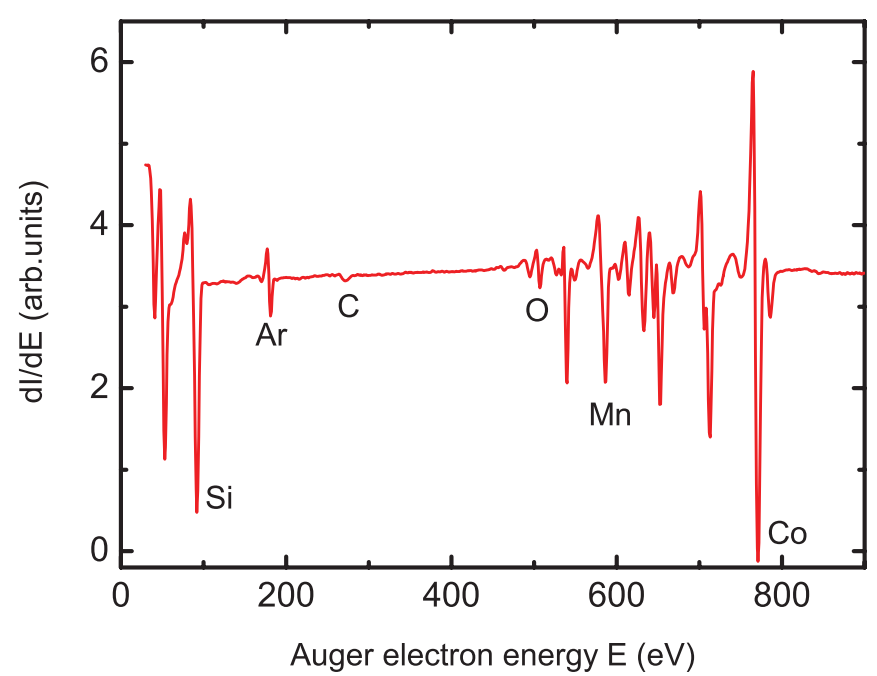

FIG. 2. (Color online) Auger spectrum of the free CMS069 (100) surface, obtained at $3 \mathrm{keV}$ primary electron energy.

\section{ELECTRONIC STRUCTURE}

\section{A. SR-PES spectra}

In Figs. 3 and 4, we show the spin-resolved photoemission spectra obtained from the freshly prepared CMS069 and CMS119 surfaces, respectively, using $p$ - and $s$-polarized laser pulses for excitation. As expected from the chemical and structural similarity, both samples show similar spectral features, labeled with $\mathrm{M} 1, \ldots, \mathrm{M} 3(\mathrm{~m} 1, \ldots, \mathrm{m} 3)$ in the majority (minority) spectra. However, the resulting electron spin polarization (ESP) shows pronounced differences in its features, labeled $(\mathrm{A}), \ldots,(\mathrm{E})$.

The most striking differences between CMS069 and CMS1 19 are observed at feature $\mathrm{A}$ in the energy region around the Fermi level. In CMS069, a negative ESP is observed for

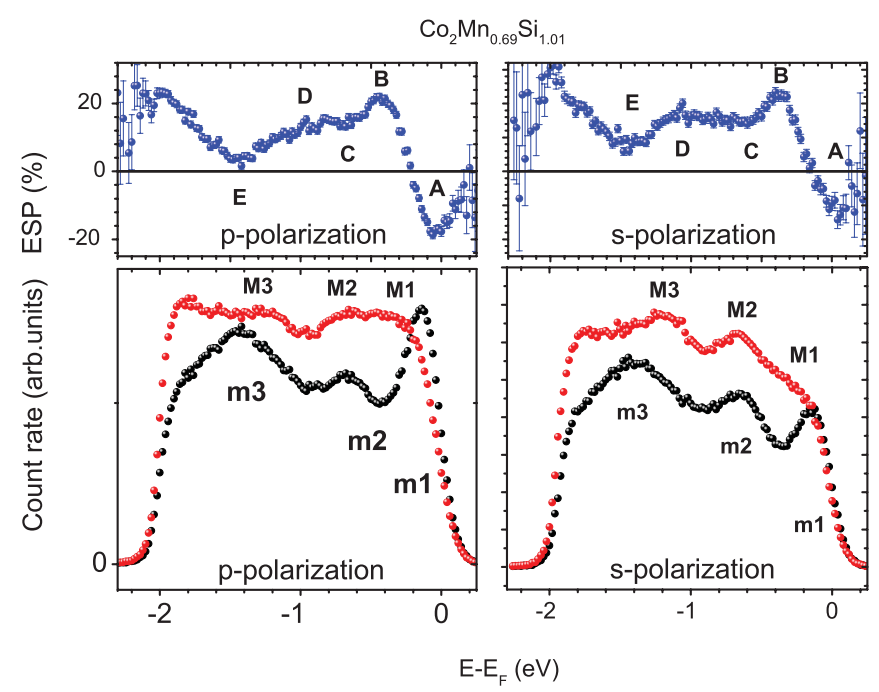

FIG. 3. (Color online) Spin-resolved photoemission spectra of the free CMS069 (100) surface for $p$-polarized excitation (left) and $s$-polarized excitation (right). Upper panel: electron spin polarization (ESP), lower panel: majority and minority count rates. ESP features are marked by A,..., E, majority (minority) features are labeled $\mathrm{M} 1, \ldots, \mathrm{M} 3(\mathrm{~m} 1, \ldots, \mathrm{m} 3)$.

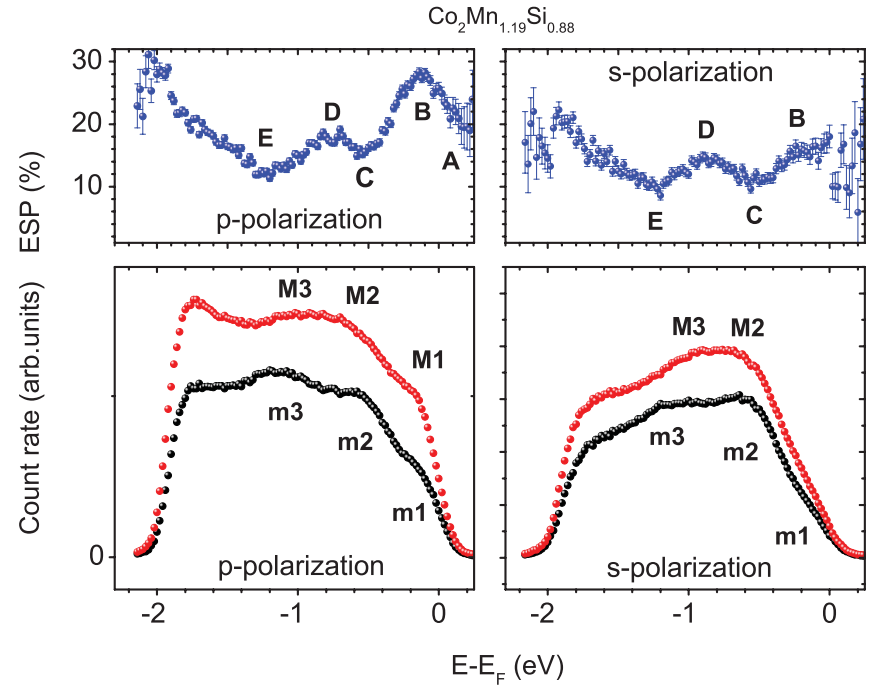

FIG. 4. (Color online) Spin-resolved photoemission spectra of the free CMS119 (100) surface for $p$-polarized excitation (left) and $s$-polarized excitation (right). Upper panel: electron spin polarization (ESP), lower panel: majority and minority count rates. ESP features are marked by A,..., E, majority (minority) features are labeled $\mathrm{M} 1, \ldots, \mathrm{M} 3(\mathrm{~m} 1, \ldots, \mathrm{m} 3)$.

both $s$ - and $p$-polarized excitations, while in CMS119, the ESP in $\mathrm{A}$ is positive for both light polarizations, but still far from $100 \%$.

Toward lower energies, the ESP recovers to values between +0.2 and +0.3 (B). Continuing further downward in energy, we observe a slight minimum $(\mathrm{C})$, followed by a maximum (D). The latter two features are more pronounced in CMS119 as compared to CMS069. A further minimum (E) in the ESP is visible for all samples. However, its energy is shifted by approximately $0.2 \mathrm{eV}$ toward $E_{F}$ in CMS119 (compare Table I). We do not observe any peak shifts due to a change of the light polarization.

In order to identify spectral features responsible for the observed ESP, we have plotted the majority and minority spectra in the lower panels of Figs. 3 and 4. The energetic positions of the most dominant spectral features are summarized in Table II. First, we note that the spectral peak positions do not depend much on the polarization direction of the incident photons.

As we can clearly see in Fig. 3, the negative ESP (A) of CMS069 at the Fermi energy is related to a strong peak $\mathrm{m} 1$ in the minority electron spectrum, with a higher contribution to the spectrum for $p$-polarized excitation than for $s$-polarized excitation. The recovery of the ESP (B) is reflected in a local

TABLE I. Energetic position of the described spin-polarization features given in $\mathrm{eV}$ with respect to $E_{F}$. The energetic position of the spectral features corresponds to their maximum relative intensity. The related statistical error is $\pm 0.05 \mathrm{eV}$ for all features.

\begin{tabular}{lrrrrr}
\hline \hline & $\mathrm{A}$ & $\mathrm{B}$ & $\mathrm{C}$ & $\mathrm{D}$ & $\mathrm{E}$ \\
\hline CMS069- $p$ & -0.20 & -0.45 & -0.70 & -0.95 & -1.45 \\
CMS069- $s$ & 0.00 & -0.40 & -0.65 & -1.10 & -1.45 \\
CMS119- $p$ & 0.00 & -0.15 & -0.55 & -0.75 & -1.25 \\
CMS119- $s$ & $\ldots$ & 0.00 & -0.50 & -0.90 & -1.25 \\
\hline \hline
\end{tabular}


TABLE II. Peak energies within the minority $(\mathrm{m})$ and majority (M) spectra given in $\mathrm{eV}$ with respect to $E_{F}$. The energetic position of the spectral features corresponds to their maximum relative intensity. The related statistical error is $\pm 0.1 \mathrm{eV}$ for the CMS119 peaks M2 and $\mathrm{M} 3$ and $\pm 0.05 \mathrm{eV}$ for all other peaks.

\begin{tabular}{lcccccc}
\hline \hline & $\mathrm{m} 1$ & $\mathrm{~m} 2$ & $\mathrm{~m} 3$ & $\mathrm{M} 1$ & $\mathrm{M} 2$ & $\mathrm{M} 3$ \\
\hline CMS069-p & -0.15 & -0.70 & -1.45 & -0.20 & -0.65 & -1.30 \\
CMS069-s & -0.15 & -0.65 & -1.40 & -0.20 & -0.70 & -1.20 \\
CMS119-p & -0.20 & -0.60 & -1.15 & -0.20 & -0.70 & -0.90 \\
CMS119-s & -0.20 & -0.65 & -1.15 & - & -0.60 & -0.90 \\
\hline \hline
\end{tabular}

minimum of minority intensity following the minority peak at the Fermi energy.

In sample CMS119, the spectral features around $E_{F}$ are less pronounced than for CMS069 but nevertheless show a clear polarization dependence (see Fig. 4). In contrast to CMS069, the ESP at $E_{F}$ is dominated by the majority spin channel. For $p$-polarized light, the majority DOS is clearly metallic, leading to the strong maximum (B). For $s$-polarized excitation, the maximum (B) is less pronounced, which is reflected in a less well defined Fermi level cutoff for majority electrons. The minority DOS around $E_{F}$ is strongly reduced as compared to CMS069. The ESP minimum (C) is formed by the features $\mathrm{m} 2$ and $\mathrm{M} 2$ in both minority and majority spectra, with similar peak positions in both samples. The small ESP increase at the low energy end of the plateau is caused by the broad majority peak M3. The latter and the minority peak $\mathrm{m} 3$ in CMS119 are both shifted by almost the same amount of about $0.2 \mathrm{eV}$ toward $E_{F}$ with respect to CMS069, causing the observed shift of the ESP minimum (E). Please note that the peaks M2 and M3 found in the CMS119 spectra are not well separated. This complicates the determination of the energetic positions, leading to a larger uncertainty of $\pm 0.1 \mathrm{eV}$. However, the assumption of only one peak in this energy region would lead in both light polarization cases to a spectral width of more than $1 \mathrm{eV}$. Such a feature is not consistent with the theoretical data presented in this work.

\section{B. Initial state symmetries}

In this section, we identify the symmetry properties of the initial states, giving rise to the intensity differences observed for different light polarization directions ( $p$ and $s)$. To this end, we have determined the $p s$-asymmetry spectra $A_{p s}=\left(I_{p}-I_{s}\right) /\left(I_{p}+I_{s}\right)$ for majority and minority electrons of each sample (see Fig. 5). Here, $I_{p}$ and $I_{s}$ are the experimental intensities of the respective photon polarization. To compensate for the larger photoelectron yield when using $p$-polarized light, the intensities of the spin-resolved spectra have been normalized to the intensity of the minority peak $\mathrm{m} 2$ for $p$ - and $s$-polarized excitation, respectively. If both polarization directions would address only states with the same symmetry, the $p s$ asymmetry would be a constant, independent of the normalization.

The observed ps asymmetry is caused by matrix element effects due to the different spatial symmetry properties of the involved initial states, and indicates the reflection symmetry of the initial states with respect to a mirror plane containing

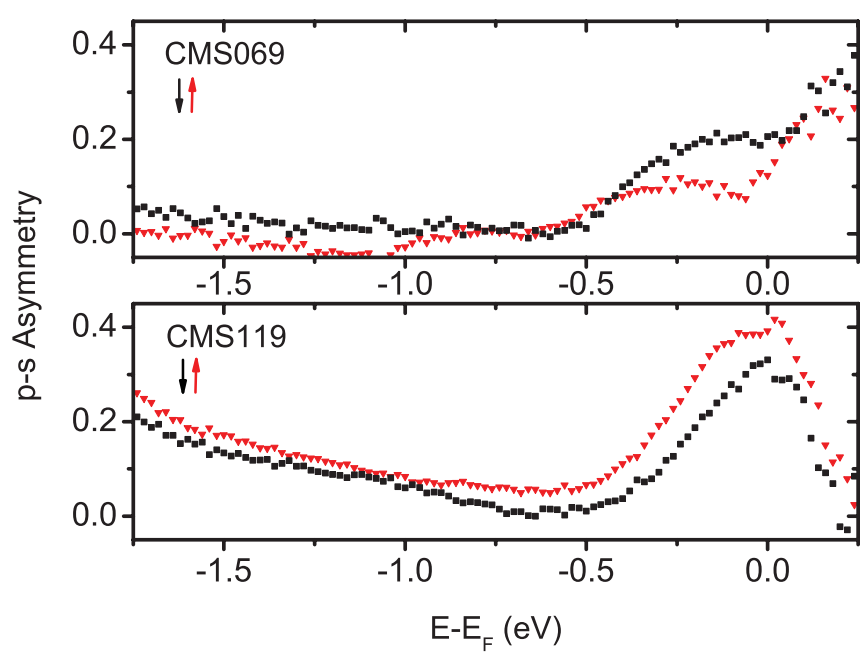

FIG. 5. (Color online) Determined experimental asymmetry spectra for $p$ - and $s$-polarized excitation in CMS069 (upper panel) and CMS119 (lower panel). Majority (minority) spin spectra are marked with red triangles (black squares). The spin-resolved spectra of each sample and polarization were normalized on the respective minority $\mathrm{m} 2$ peak.

the sample surface normal. While $s$-polarized light has only components along the in-plane [011] direction and thus only excites states with $\Delta_{5}$ symmetry, $p$-polarized light additionally excites states of $\Delta_{1}$ symmetry in our experimental geometry, due to its field component along the normal [100] direction. ${ }^{49}$ The variation of the $p s$-asymmetry spectrum thus can be used as a measure for the contribution of states compatible with $\Delta_{1}$ symmetry, be it a bulk, defect, or a surface related transition. The background is formed by a common contribution originating from $\Delta_{5}$ initial states that contribute to the spectrum of both light polarization directions.

The experimental ps asymmetry of majority (M) and minority (m) electron spectra for CMS069 and CMS119 are displayed in Fig. 5. We observe a strong $p s$-asymmetry maximum around the Fermi energy $E_{F}$ in both samples, for both majority and minority electrons. This asymmetry peak extends down to $-0.6 \mathrm{eV}$, with its maximum situated close to the features M1 and $\mathrm{m} 1$. We observe a clear substructure in CMS069, which can be related to the shift between the center of gravity of the peak M1 and the sharp peak $\mathrm{m} 1$. A relative minimum is reached close to the position of the features $\mathrm{m} 2$ and M2. Toward lower energies, the $\Delta_{1}$ contribution stays stable (CMS069) or rises slightly (CMS119). In contrast to CMS069, the $\Delta_{1}$ contribution in CMS119 is stronger in the majority spectrum for CMS119, leading to an increase of the measured ESP slightly below $E_{F}$ in the Mn-rich sample and for $p$-polarized light.

\section{Resonant bulk transitions}

In this section, we identify the possible contributions of bulk electronic transitions and final-state effects to the experimental spectra presented in Sec. V A. For this purpose, we calculated the bulk spectral functions of stoichiometric CMS along the surface normal using LSDA + DMFT. In Fig. 6, we show an overlay of the ideal $L 2_{1}$ occupied bands (black) and a 


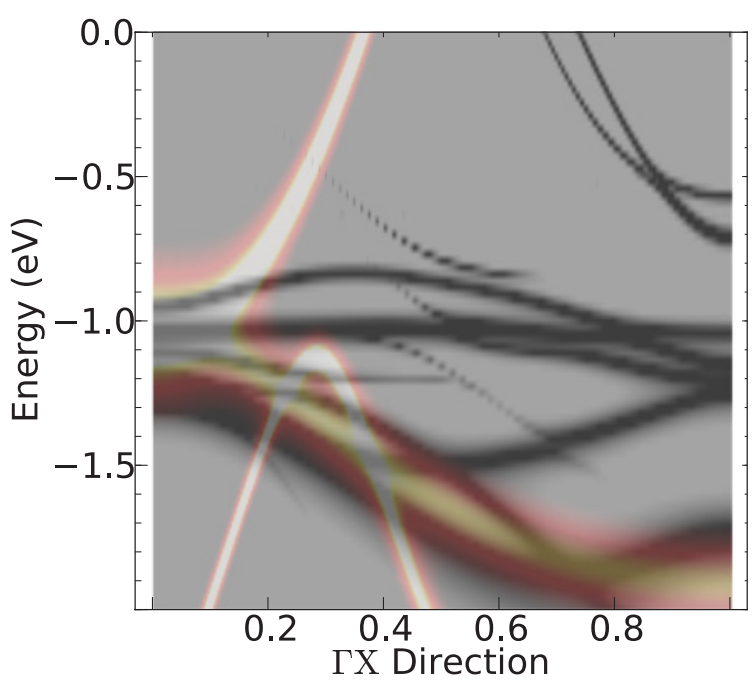

(a) Majority

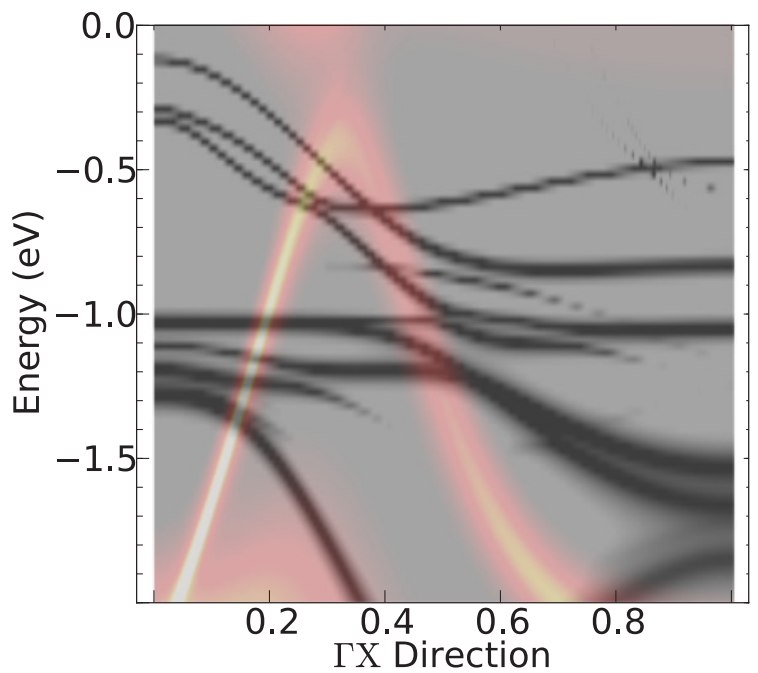

(b) Minority

FIG. 6. (Color online) Possible bulk transitions for majority and minority electrons along the $\Gamma \mathrm{X}$ momentum direction of CMS as calculated using LSDA + DMFT. Occupied bulk states (unoccupied final states) are shown as black (orange) lines. Crossing points correspond to energy- and momentum-allowed transitions at the photon energy of $\hbar \omega=5.9 \mathrm{eV}$.

replica (orange) of the unoccupied final states, where the latter are shifted downward by the experimental photon energy of $\hbar \omega=5.9 \mathrm{eV}$. In this way, energy- $(\Delta E=\hbar \omega)$ and momentumallowed $(\Delta \mathbf{k}=0)$ bulk resonant interband transitions appear as crossing points between occupied and unoccupied bands and can directly be compared to the energies of measured spectral features. Matrix-element effects such as symmetry selection rules are not considered in this picture. Since the momentum conservation is broken along the surface normal, all transitions along the $\Gamma X$ direction are expected to contribute to the measured photoelectron spectrum.

First, we note that there are no allowed resonant bulk majority transitions in the vicinity of the Fermi energy. Resonant bulk transitions in the majority spin direction can only be found below $E-E_{F}=-0.9 \mathrm{eV}$. They involve localized orbitals as initial states and most probably constitute the main contribution to the broad majority M3 peak. Above this energy, we find dispersive bulk majority electron states with $\Delta_{1}$ and $\Delta_{5}$ symmetry, ${ }^{50}$ with their band bottom situated 0.6 and $0.7 \mathrm{eV}$ below the Fermi energy, respectively. These states might be the origin of surface resonances that form the complex peak consisting of M1 and M2 in the majority spectra, and thus might contribute significantly to the measured ESP in the vicinity of $E_{F}$.

For minority electrons, possible resonant bulk transitions can be found starting at $0.5 \mathrm{eV}$ below the bulk valence band maximum. These transitions stem from the dispersive minority valence band and contribute to the minority peak $\mathrm{m} 2$ in the spectra. Between -1.0 and $-1.4 \mathrm{eV}$ a second, more localized set of resonant transitions appears, which we assign to the positions of peak $\mathrm{m} 3$ in the minority spectra. Due to their flat dispersion, these states should be robust against final-state effects.

In the vicinity of the Fermi energy, only nonresonant optical minority electron transitions are possible, and no intensity should be expected from the minority gap region due to the lack of initial states. Thus, at our photon energy, the measured ESP around the Fermi energy should depend sensitively on additional gap states caused by bulk defects or by the surface. These states are thus the dominant contribution to the observed minority peak $\mathrm{m} 1$, as will be discussed in Secs. V D and V E.

\section{Disorder effects for $\alpha=\mathbf{0 . 6 9}$ and 1.19}

In order to identify possible bulk disorder effects that might affect the measured ESP, we have calculated the spinresolved density of states (DOS) for the nominal compositions $\mathrm{Co}_{2} \mathrm{Mn}_{\alpha} \mathrm{Si}$ with $\alpha=0.69$ and 1.19, using LSDA + CPA. Here, we restrict ourselves to the DOS since bulk defects are intrinsically localized and thus are not affected by final-state effects. The lattice occupations have been inferred from the formula unit model introduced by Yamamoto et al.,${ }^{27}$ assuming that the $L 2_{1}$ structure is preserved and that the formation of bulk vacancies is energetically unfavorable compared to the formation of antisites.

This assumption has first been tested using $\mathrm{Co}_{2} \mathrm{Mn}_{0.69} \mathrm{Si}$. Two limiting cases have been modeled: the vacancy model assumes that the positions of the missing $\mathrm{Mn}$ atoms are left empty, and the antisite model assumes that these positions are filled with $\mathrm{Co}$ and $\mathrm{Si}$ atoms to preserve a full occupation of the $L 2_{1}$ lattice. In Figs. 7(a) and 7(b), we show the calculated spin polarization and spin-resolved DOS for the antisite model $\mathrm{Co}_{2}\left(\mathrm{Co}_{0.168} \mathrm{Mn}_{0.748} \mathrm{Si}_{0.084}\right) \mathrm{Si}$ and the vacancy model $\mathrm{Co}_{2}\left(\mathrm{Mn}_{0.69}\right.$ Vacancy $\left._{0.31}\right) \mathrm{Si}$, respectively.

Surprisingly, the antisite model reproduces almost all features $(\mathrm{A}, \ldots, \mathrm{E})$ of the measured spin polarization of CMS069. Nevertheless, for the energetic positions of features $D$ and E, one finds some discrepancy. Compared to the pure LSDA calculations for stoichiometric CMS (taken from Ref. 5 and marked by dashed lines), the minority gap at the Fermi energy is closed in the antisite model. The additional electrons due to the excess Co are thus mainly accommodated in minority gap states. In addition, the maximum in the majority DOS appears slightly shifted toward $E_{F}$ in the antisite model DOS.

There are two particularities of the spectra we want to highlight. First, LSDA + CPA underestimates the measured 

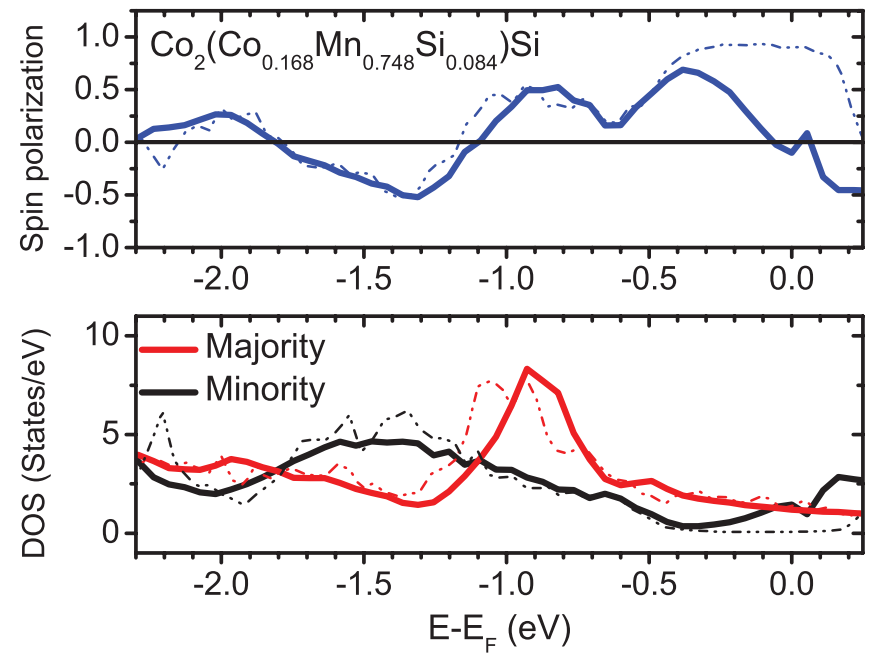

(a) Antisite
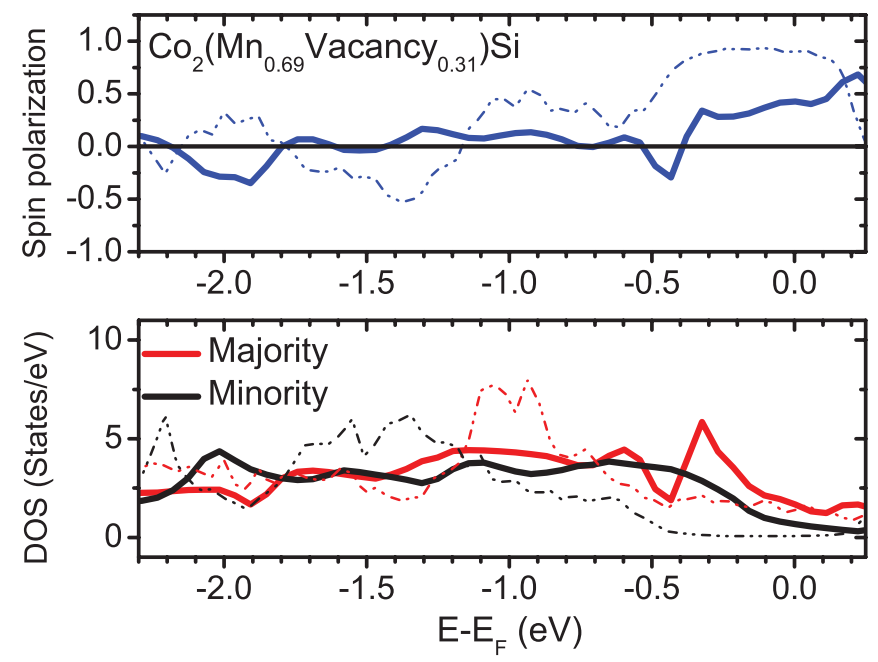

(b) Vacancy

FIG. 7. (Color online) CPA calculations for $\alpha=0.69$. Missing $\mathrm{Mn}$ atoms are replaced by (a) $\mathrm{Co}$ and $\mathrm{Si}$ atoms and (b) vacancies. Upper panel: spin polarization. Lower panel: majority (red) and minority (black) DOS. Dashed lines represent pure LSDA calculations of stoichiometric CMS as published in Ref. 5.

spin polarization in CMS069, predicting a Fermi level ESP of $-10 \%$ instead of the measured $-20 \%$. This is astonishing since calculations tend to predict larger absolute ESP values than observed in spectra, and points to an additional surface effect that is not considered in the LSDA + CPA calculations. A second aspect is related to the positions of the main peaks in the minority and majority DOS. While the minority peak at $-1.44 \mathrm{eV}$ fits perfectly to the peak $\mathrm{m} 3$ of the experimental CMS069 spectrum, the strong majority DOS peak at $-0.92 \mathrm{eV}$ has no direct counterpart in the measured majority spectrum of CMS069. This points out the relevance of final-state effects for the bulk related features of the spectra. Apart from that, it is obvious that the formula unit model agrees well with our spectra, indicating a large contribution of bulk $\mathrm{Co}_{\mathrm{Mn}}$ antisite defect states that contribute to the $\mathrm{m} 1$ feature in the minority bands of the Mn-deficient CMS069 sample.
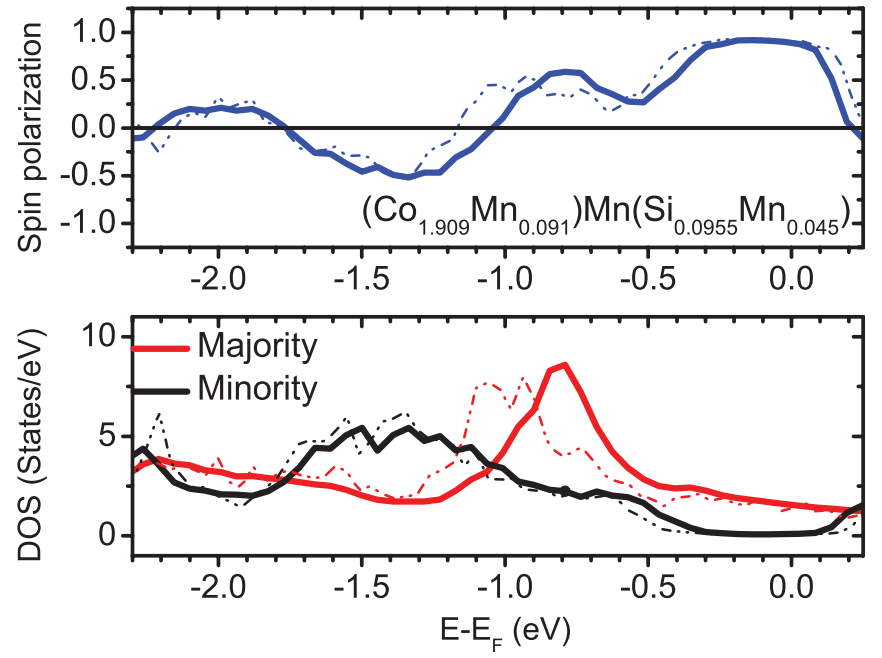

FIG. 8. (Color online) CPA calculations for $\alpha=1.19$. Surplus $\mathrm{Mn}$ atoms are hosted by the $\mathrm{Co}$ and $\mathrm{Si}$ sublattice. Upper panel: spin polarization. Lower panel: majority (red) and minority (black) DOS. Dashed lines represent LSDA calculations of stoichiometric CMS.

In contrast to the antisite model, the introduction of a large degree of vacancies into the $L 2_{1}$ structure results in a massive change of the electronic structure both in the majority and the minority DOS that does not correlate with the experimental data and thus will be considered no further.

We will now focus on the Mn-rich sample CMS119. According to Ref. 27, increasing the Mn to Co ratio even above the stoichiometric $1: 2$ should suppress the detrimental $\mathrm{Co}_{\mathrm{Mn}}$ antisites and lead to an improved ESP. We calculated the spin polarization and spin-resolved DOS for the Mn-rich $\mathrm{Co}_{2} \mathrm{Mn}_{1.19} \mathrm{Si}$ using the composition $\left(\mathrm{Co}_{1.909} \mathrm{Mn}_{0.091}\right) \mathrm{Mn}\left(\mathrm{Si}_{0.955} \mathrm{Mn}_{0.045}\right)$ according to the formula unit model (see Fig. 8). Most importantly, the minority spin gap remains open in this configuration, though its width is slightly reduced.

As in CMS069, the energetic positions of the ESP features $\mathrm{B}$ and $\mathrm{C}$ are almost exactly reproduced. The predicted position of $\mathrm{E}$ is the same as for CMS069, but the corresponding experimental CMS 119 minority feature $\mathrm{m} 3$ is shifted by $0.2 \mathrm{eV}$ toward $E_{F}$ (cf. Table II). This might be a final-state effect or a surface effect. As already observed for the Mn-deficient case, the maximum of the majority DOS appears shifted to $E=-0.8 \mathrm{eV}$, coinciding roughly with the majority feature M3 at $E=-0.92 \mathrm{eV}$ of CMS119. This might be a consequence of $\mathrm{Mn}_{\mathrm{Co}}$ antisites, which reduce the number of electrons in the majority bands. Otherwise the DOS is nearly identical to the one of stoichiometric CMS. The experimental spin polarization is qualitatively well reproduced by the simulation, but in contrast to CMS069 the calculations yield much higher absolute values of the ESP. We obtain a large positive spin polarization at the Fermi energy, which confirms the interpretation that $\mathrm{Mn}_{\mathrm{Co}}$ and $\mathrm{Mn}_{\mathrm{Si}}$ antisites do not affect the half-metallic properties for $\alpha>1$ at $E_{F}$. The slight decrease in the measured ESP close to the Fermi energy for $p$-polarized light is not reproduced. As an explanation, we propose that either the disorder induced narrowing of the minority band 


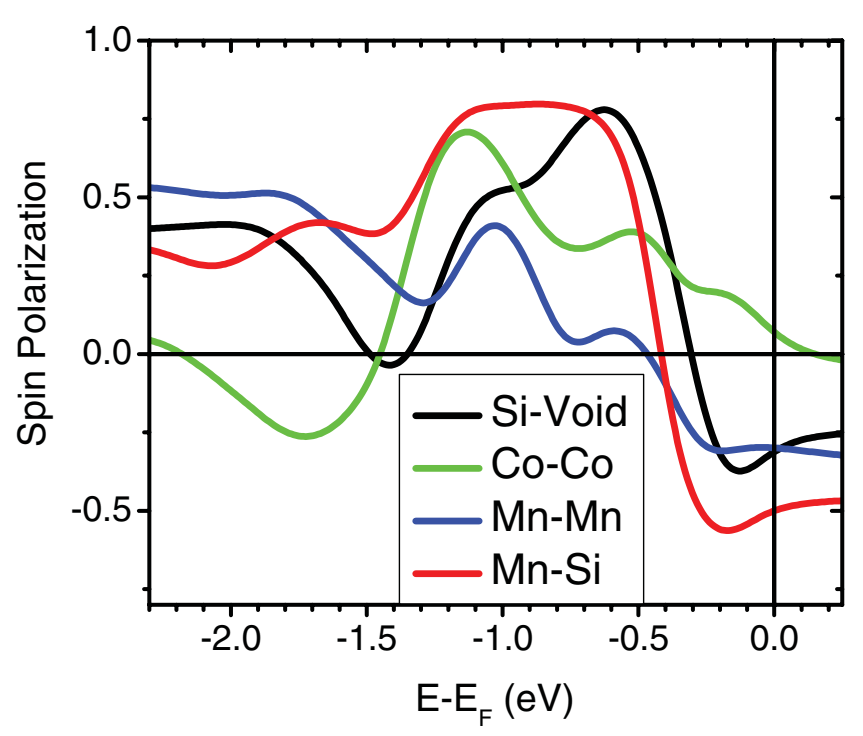

FIG. 9. (Color online) Calculated spin-polarization spectra of the CMS(100) surface, using a photon energy of $5.9 \mathrm{eV}$ and $s$-polarized light.

gap or the presence of minority surface states are responsible for the ESP reduction very close to $E_{F}$.

\section{E. Surface effects}

As we have seen in the previous sections, several spectral features can not be fully explained neither by bulk transitions nor by defects. Therefore possible surface effects will be taken into account in the following. In order to illustrate the sensitivity of the spin polarization to the surface termination, we calculated spin-resolved photoemission spectra for all thermodynamically stable surface terminations of stoichiometric $\mathrm{CMS},{ }^{30}$ using LSDA + CPA + DMFT in the framework of the one-step model of photoemission.
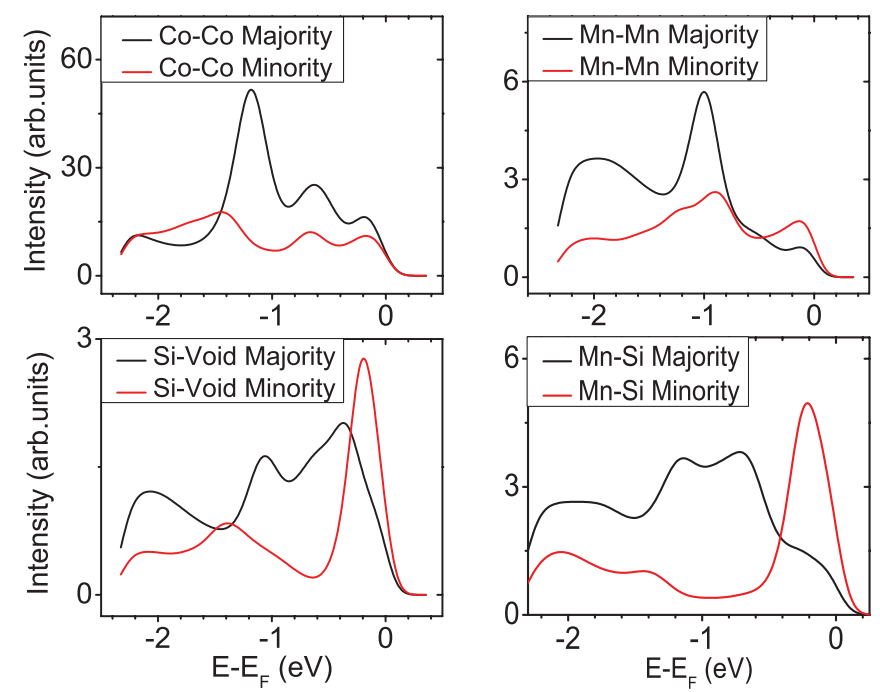

FIG. 10. (Color online) Calculated majority and minority spectra of the CMS(100) surface, terminated by a final Co-Co, Mn-Mn, Sivoid, and $\mathrm{Mn}-\mathrm{Si}$ layer, and using a photon energy of $5.9 \mathrm{eV}$ and $s$-polarized light.
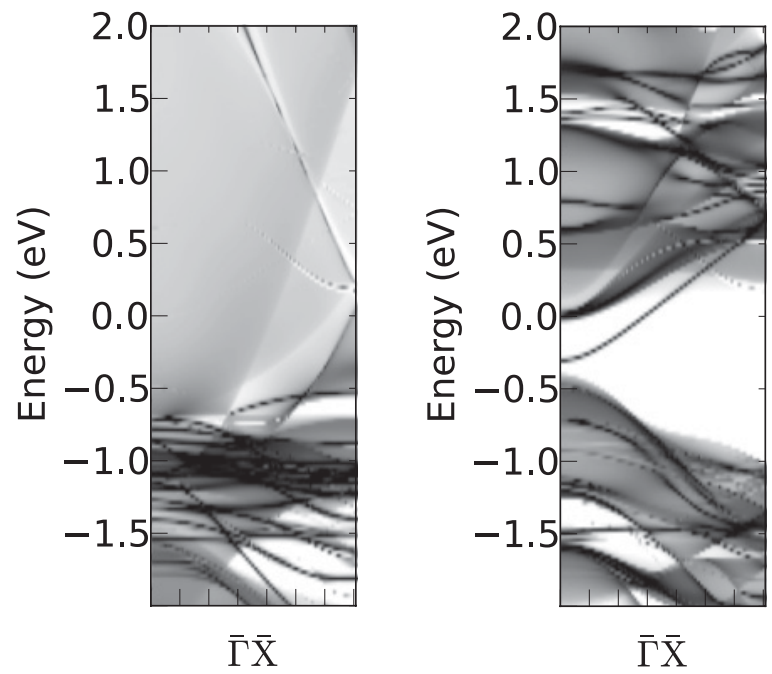

FIG. 11. Surface electronic structure of the Mn-Mn terminated CMS surface along the $\bar{\Gamma} \bar{X}$ direction, calculated in the LSDA scheme. Left panel: majority electrons. Right panel: minority electrons.

Figure 9 shows the spin polarization calculated for the Co$\mathrm{Co}$, the $\mathrm{Mn}-\mathrm{Si}$, the $\mathrm{Mn}-\mathrm{Mn}$ and the vacancy-Si terminations. The corresponding calculated spin-resolved spectra are shown in Fig. 10. All surface terminations show a strong reduction of the spin polarization at the Fermi energy, i.e., none of the sample surfaces is truly half-metallic in our calculation. This holds in particular for the Mn-Mn terminated surface, which has earlier been suggested to be half-metallic. ${ }^{30}$ In our calculations for that surface termination, we clearly observe a dispersive minority surface state along $\Gamma X$ that crosses the Fermi energy (cf. Fig. 11).

Let us first compare the results of the calculations to the measured spin polarization of CMS069 (see Fig. 3). For the vacancy-Si and the $\mathrm{Mn}-\mathrm{Si}$ terminations corresponding to the observed LEED patters, we find a good qualitative agreement with the experimental ESPs, in particular with the features B to E. Moreover, the negative spin polarization at the Fermi energy as well as the energetic positions of the spin polarization features $\mathrm{D}$ and $\mathrm{E}$ are even better reproduced than in the CPA calculations. The monoatomic Co-Co and Mn-Mn terminations are less similar to the measured spinpolarization spectra, emphasizing the strong impact of the surface termination on the photoemission spectra.

In CMS119, we found that the influence of surface states on the experimental data is less than predicted in the calculations based on stoichiometric CMS, even though CMS119 should be electronically more similar to bulk CMS than CMS069. Although peaks M1 and $\mathrm{m} 1$ show strong dependence on the light polarization and almost vanish for $s$-polarized light, none of the calculated photoemission spectra (see Fig. 10) show acceptable agreement with the experimental data. Therefore we conclude that this behavior reflects the presence of bulk states with predominant $\Delta_{1}$ symmetry at this energy.

\section{CONCLUSIONS}

We investigated the free surface of thin $\mathrm{Co}_{2} \mathrm{Mn}_{\alpha} \mathrm{Si}(100)$ films using a combination of LEED, spin-resolved photoelectron spectroscopy, and electronic structure calculations. Due to 
the low dispersion of the bulk $d$ bands of CMS, many features of the experimental spectra can directly be related to bulk features. However, in particular, in the range of the Fermi energy where the majority bands are strongly dispersive, resonant majority interband transitions are momentum-forbidden for the selected photon energy, rendering our experiment sensitive to even small amounts of defects or surface states not only in the minority but also in the majority bands. The experimental spin-resolved photoemission spectra of the free surfaces of nonstoichiometric $\mathrm{Co}_{2} \mathrm{MnSi}$ films are in good agreement with LSDA + CPA calculations based on the formula unit model that assumes the formation of antisites rather than vacancies for nonstoichiometric $\mathrm{Co}_{2} \mathrm{MnSi}$. Explicit photoemission calculations indicate that the surface termination also has a strong influence on the surface electronic spin polarization. Our experimental spin-resolved photoelectron spectra enable us to trace back the observed spectral features, finding bulk states at lower energies as well as surfaceand defect-induced states close to $E_{F}$. For the Mn-deficient compound, best agreement is obtained for both the $\mathrm{Mn}-\mathrm{Si}$ and vacancy-Si terminated surfaces (or a mixture of both, caused by the nonstoichiometric sample composition), which is also supported by the LEED patterns. Polarization-dependent measurements indicate majority as well as minority states with a strong $\Delta_{1}$-compatible symmetry contribution at the Fermi energy. Here, the negative spin polarization at $E_{F}$ is related to minority surface and bulk antisite defect states, which is also consistent with the formula unit model. In the Mn-rich compound, we find no sign of bulk defect induced minority gap states or surface states. Here, the spin polarization at $E_{F}$ is mainly determined by the contribution of majority electrons with $\Delta_{1}$ symmetry.

\section{ACKNOWLEDGMENTS}

Financial support through the DFG Research Unit 1464 ASPIMATT and FOR 1346, by the German ministry BMBF under contract $05 \mathrm{KS} 10 \mathrm{WMA}$ is gratefully acknowledged. The work at Hokkaido University was partly supported by a Grantin-Aid for Scientific Research (A) (Grant No. 23246055) from MEXT, Japan. We thank M. Jourdan and C. Herbort for providing us a CCFA sample for the comparison of the LEED patterns. *jpwuest@physik.uni-kl.de

${ }^{1}$ B. Balke, S. Wurmehl, G. Fecher, C. Felser, and J. Kübler, Sci. Techn. Adv. Mat. 9, 014102 (2008).

${ }^{2}$ T. Ishikawa, H. xi Liu, T. Taira, K. ichi Matsuda, T. Uemura, and M. Yamamoto, Appl. Phys. Lett. 95, 232512 (2009).

${ }^{3}$ Y. Sakuraba, N. Hirose, M. Oogane, T. Nakamura, Y. Ando, and K. Takanashi, Appl. Phys. Lett. 96, 092511 (2010).

${ }^{4}$ S. Ishida, S. Fujii, S. Kashiwagi, and S. Asano, J. Phys. Soc. Jpn. 64, 2152 (1995).

${ }^{5}$ S. Chadov, G. Fecher, C. Felser, J. Minar, J. Braun, and H. Ebert, J. Phys. D: Appl. Phys. 42, 084002 (2009).

${ }^{6}$ L. Chioncel, Y. Sakuraba, E. Arrigoni, M. I. Katsnelson, M. Oogane, Y. Ando, T. Miyazaki, E. Burzo, and A. I. Lichtenstein, Phys. Rev. Lett. 100, 086402 (2008).

${ }^{7}$ P. Webster, J. Phys. Chem. Solids 32, 1221 (1971).

${ }^{8}$ Y. Sakuraba, M. Hattori, M. Oogane, Y. Ando, H. Kato, A. Sakuma, T. Miyazaki, and H. Kubota, Appl. Phys. Lett. 88, 192508 (2006).

${ }^{9}$ T. Ishikawa, N. Itabashi, T. Taira, K. Matsuda, T. Uemura, and M. Yamamoto, J. Appl. Phys. 105, 07B110 (2009).

${ }^{10}$ M. Jullière, Phys. Lett. A 54, 225 (1975).

${ }^{11}$ A. Rajanikanth, Y. K. Takahashi, and K. Hono, J. Appl. Phys. 105, 063916 (2009).

${ }^{12}$ W. Wang, M. Przybylski, W. Kuch, L. Chelaru, J. Wang, Y. Lu, J. Barthel, and J. Kirschner, J. Magn. Magn. Mater. 286, 336 (2005).

${ }^{13}$ H. Schneider, G. Jakob, M. Kallmayer, H. J. Elmers, M. Cinchetti, B. Balke, S. Wurmehl, C. Felser, M. Aeschlimann, and H. Adrian, Phys. Rev. B 74, 174426 (2006).

${ }^{14}$ M. Cinchetti, J.-P. Wüstenberg, M. Sanchez Albaneda, F. Steeb, A. Conca, M. Jourdan, and M. Aeschlimann, J. Phys. D: Appl. Phys. 40, 1544 (2007).

${ }^{15}$ J.-P. Wüstenberg, M. Cinchetti, M. S. Albaneda, M. Bauer, and M. Aeschlimann, J. Magn. Magn. Mater. 316, E411 (2007).

${ }^{16}$ J.-P. Wüstenberg, J. Fischer, C. Herbort, M. Jourdan, M. Aeschlimann, and M. Cinchetti, J. Phys. D: Appl. Phys. 42, 084016 (2009).
${ }^{17}$ M. Hahn, G. Schönhense, E. A. Jorge, and M. Jourdan, Appl. Phys. Lett. 98, 232503 (2011).

${ }^{18}$ V. Irkhin, M. Katsnelson, and A. Lichtenstein, J. Phys. Condens. Matter 19, 315201 (2007).

${ }^{19}$ G. H. Fecher, B. Balke, A. Gloskowskii, S. Ouardi, C. Felser, T. Ishikawa, M. Yamamoto, Y. Yamashita, H. Yoshikawa, S. Ueda, and K. Kobayashi, Appl. Phys. Lett. 92, 193513 (2008).

${ }^{20}$ K. Miyamoto, A. Kimura, Y. Miura, M. Shirai, M. Ye, Y. Cui, K. Shimada, H. Namatame, M. Taniguchi, Y. Takeda, Y. Saitoh, E. Ikenaga, S. Ueda, K. Kobayashi, and T. Kanomata, Phys. Rev. B 79, 100405(R) (2009).

${ }^{21}$ P. Mavropoulos, K. Sato, R. Zeller, P. H. Dederichs, V. Popescu, and H. Ebert, Phys. Rev. B 69, 054424 (2004).

${ }^{22}$ M. Ležaić, P. Mavropoulos, J. Enkovaara, G. Bihlmayer, and S. Blügel, Phys. Rev. Lett. 97, 026404 (2006).

${ }^{23}$ R. Skomski, J. Phys. Condens. Matter 19, 315202 (2007).

${ }^{24}$ S. Picozzi, A. Continenza, and A. J. Freeman, Phys. Rev. B 69, 094423 (2004).

${ }^{25}$ I. Galanakis, K. Özdoğan, B. Aktaş, and E. Şaşioğlu, Appl. Phys. Lett. 89, 042502 (2006).

${ }^{26}$ B. Hülsen, M. Scheffler, and P. Kratzer, Phys. Rev. B 79, 094407 (2009).

${ }^{27}$ M. Yamamoto, T. Ishikawa, T. Taira, G.-f. Li, K. Matsuda, and T. Uemura, J. Phys. Condens. Matter 22, 164212 (2010).

${ }^{28}$ H. Lüth, Solid Surfaces, Interfaces and ThinFilms (Springer-Verlag, Heidelberg, Berlin, New York, 2001).

${ }^{29}$ P. Mavropoulos, M. Ležaić, and S. Blügel, Phys. Rev. B 72, 174428 (2005).

${ }^{30}$ S. J. Hashemifar, P. Kratzer, and M. Scheffler, Phys. Rev. Lett. 94, 096402 (2005)

${ }^{31}$ H. Ebert, D. Ködderitzsch, and J. Minár, Rep. Prog. Phys. 74, 096501 (2011).

${ }^{32}$ H. Ebert, in Electronic Structure and Physical Properties of Solids, edited by H. Dreyssé, Lecture Notes in Physics Vol. 535 (Springer, Berlin, 2000), p. 191. 
${ }^{33}$ The Munich SPR-KKR package, version 5.4, H. Ebert et al., [http://olymp.cup.uni-muenchen.de/ak/ebert/SPRKKR] (2009).

${ }^{34}$ S. H. Vosko, L. Wilk, and M. Nusair, Can. J. Phys. 58, 1200 (1980).

${ }^{35}$ J. Minár, J. Phys. Condens. Matter 23, 253201 (2011).

${ }^{36}$ J. Minár, L. Chioncel, A. Perlov, H. Ebert, M. I. Katsnelson, and A. I. Lichtenstein, Phys. Rev. B 72, 045125 (2005).

${ }^{37}$ M. I. Katsnelson and A. I. Lichtenstein, Eur. Phys. J. B 30, 9 (2002).

${ }^{38}$ L. V. Pourovskii, M. I. Katsnelson, and A. I. Lichtenstein, Phys. Rev. B 73, 060506 (2006).

${ }^{39}$ S. Chadov, J. Minár, M. I. Katsnelson, H. Ebert, D. Ködderitzsch, and A. I. Lichtenstein, Europhys. Lett. 82, 37001 (2008).

${ }^{40}$ O. Šipr, J. Minár, S. Mankovsky, and H. Ebert, Phys. Rev. B 78, 144403 (2008).

${ }^{41}$ J. Braun, J. Minár, F. Matthes, C. M. Schneider, and H. Ebert, Phys. Rev. B 82, 024411 (2010).

${ }^{42}$ J. Minár, J. Braun, S. Mankovsky, and H. Ebert, J. Electron Spectrosc. Relat. Phenom. 184, 91 (2011).
${ }^{43} \mathrm{G}$. Ertl and J. Küppers, Low Energy Electrons and Surface Chemistry (VCH Wiley, Weinsheim, 1985).

${ }^{44}$ L. Davis, N. MacDonald, P. Palmberg, and G. Riach, Handbook of Auger Electron Spectroscopy: A Reference Book of Standard Data for Identification and Interpretation of Auger Electron Spectroscopy Data (Physical Electronics, Minnesota, 1987).

${ }^{45}$ J. Kirschner, Polarized Electrons at Surfaces (Springer-Verlag, Berlin, Heidelberg, New York, Tokyo, 1985).

${ }^{46}$ C. Herbort, E. Arbelo, and M. Jourdan, J. Phys. D: Appl. Phys. 42, 084006 (2009).

${ }^{47}$ T. Miyajima, M. Oogane, Y. Kotaka, T. Yamazaki, M. Tsukada, Y. Kataoka, H. Naganuma, and Y. Ando, Appl. Phys. Express 2, 093001 (2009).

${ }^{48}$ Surface Analysis by Auger and X-Ray Photoelectron Spectroscopy, edited by D. Briggs and J. Grant (IM Publications and SurfaceSpectra Limited, Manchester, 2003).

${ }^{49}$ J. Hermanson, Solid State Commun. 22, 9 (1977).

${ }^{50}$ Y. Miura, H. Uchida, Y. Oba, K. Nagao, and M. Shirai, J. Phys. Condens. Matter 19, 365228 (2007). 